\title{
THE DISTRIBUTION AND STRUCTURE OF THE MICROVASCULATURE OF THE PHALLUS IN THE OSTRICH (Struthio camelus)
}

\author{
Elias, M. Z. J. ${ }^{1}$, Petrovová, E. ${ }^{2}$, Vdoviaková, K. ${ }^{2}$ \\ Maloveská, M. ${ }^{2}$, Teleky, J. ${ }^{2}$, Krešáková, L. ${ }^{2}$ \\ ${ }^{1}$ Faculty of Veterinary Medicine, Eduardo Mondlane University, Maputo \\ Mozambique \\ ${ }^{2}$ Department of Anatomy, Histology and Physiology \\ University of Veterinary Medicine and Pharmacy, Komenského 73, 04181 Košice \\ The Slovak Republic \\ lenka.kresakova@uvlf.sk
}

\section{ABSTRACT}

This study determined the distribution and specialized morphology (gaps and fenestrations, irregular endothelium, internalization of luminal material) of vessels in the spongy and erectile tissue of the phallus in the ostrich. Specimens from the phallus harvested from 18 sexual mature and active ostriches were utilized. General routine fixation was used for tissue blocks and then they were processed conventionally for observation under the Transmission Electron Microscope (TEM). The spongy tissue in the root of the phallus exhibited several blood vessels (mainly venules) within connective tissue strands. The capillaries displayed thick and attenuated endothelial profiles, whereas the venules were lined with an attenuated endothelium. Both vessel types displayed fenestrations and prominent gaps supported by a continuation of the basal lamina. Numerous cytoplasmic processes extended from the capillary endothelium, in places appearing to enclose and sequestrate luminal contents by phagocytosis. The internalized material appeared in the form of relatively large, pale vesicles. The endothelium was extremely irregular in profile, in some instances appearing to be composed of more than one layer of squamous cells. The erectile tissue lining the phallic sulcus presented relatively few capillaries and venules. These vessels exhibited features similar to those seen in the spongy tissue but the endothelial cells were markedly more complex and irregular in design.

Key words: erection mechanism; male ostrich; microvasculature; phallus; reproductive system

\section{INTRODUCTION}

The phallus is a structure formed by closely related folds of tissue that derives from the ventral wall of the proctodeum in male avian species. It is analogous to the mammal penis, because it transfers ejaculated spermatozoa to the female reproductive organs [1]. There are two types of phalluses, the intromittent (penetrates the female cloaca) and non-intromittent (phallus does not penetrate the female cloaca, male and female cloaca are simple pressed 
together to transfer sperm). The ostrich phallus is of intromittent type. The erection of the phallus was investigated by Rautenfeld [15], but this still needs more research using modern techniques [14].

The gross morphological structure of the ostrich phallus has been described $[2,5,8]$. No information is available on the microvascularization of that segment of the reproductive organs, although the gross pattern of both arterial supply and venous drainage of the male reproductive tract of the ostrich has been investigated $[3,4,18]$. The paracloacal vascular body (corpus vasculare paracloacale) is the source of lymph that erects the phallus in ducks and roosters [8]. Reports on the corpus paracloacalis vascularis of the domestic fowl $[12,19,20]$, turkey [11] and Guinea fowl [17] have been presented.

In the rooster, the paracloacal vascular body is a small, red oval structure located in the wall of urodeum close to the receptacle of the deferent duct; it is covered with a connective tissue capsule [12]. In light microscopy, the paracloacal vascular body reveals capsule, trabeculae, capillary cords and lymphatic spaces. The lymphatic spaces are divided into peripheral lymphatic spaces under the capsule and the internal lymphatic spaces in the parenchyma [19]. Lymphatic channels are present close to a group of capillaries [7]. The trabeculae have one or two arterioles and venules inside. SEM of corrosion casts reveal anastomosing vascular cords between which lay lymphatic spaces [19]. Transmission electron microscopy reveals capillaries and many pericytes in the cords with a layer of endothelial cells with rare fenestrations with diaphragm [19]. Gunawardana and Scott [7] also described fenestrations in the endothelial processes but in this instance they did not show any diaphragms. In the turkey, two units of paracloacal vascular body are located caudoventrally to the proctodeum [11]. This structure possesses large lymphatic sinuses. Several capillaries are inside the paracloacal vascular body and they are surrounded by lymph sinuses. In the turkey, the connective tissue cords also contain blood vessels and form a network that supports the intervening lymphatic spaces [11]. In the guinea fowl, the paracloacal vascular body comprises a capsule, peripheral and internal lymphatic sinuses, trabeculae and capillary cards. Arterioles and venules are present, and the capillaries display rare fenestrations [17]. For a review of the structure of the paracloacal vascular body see $[8,10]$.

The paracloacal vascular body is present in different avian species, the names given to it by different authors may differ. King [9] in Nomina Anatomica calls it "Corpus vasculare phalli", and refers to other names "Glomera corporis vasculare phalli", "Lymphobulbus phalli"; this name was used also by Rauten feld [15], "Corpus paracloacalis vascularis" $[12,19,20]$, paracloacal vascular body [17]. In the ostrich, a structure equivalent to paracloacal vascular body has not yet been established. Reports from previous authors, did not elucidate on the issue [8].

The paracloacal vascular body received attention because of the absence of accessory sexual glands in male birds and the involvement of this body in the erection of the phallus $[8,20]$. The paracloacal vascular body is believed to supply lymph to the phallus for erection in various avian species such as the fowl and the duck [8] and ostrich [15].

This study determined the distribution and structure of microvasculature in the phallus of the ostrich using India ink injection (Light Microscopy) and Transmission Electron Microscopy (TEM).

\section{MATERIALS AND METHODS}

The torsos of 18 sexually mature and active male ostriches, were obtained from the Oryx abattoir in Krugersdorp, Gauteng, in South Africa. The vascular system of the reproductive tract of six of the ostriches was flushed free of blood by injecting physiological saline through the descending aorta. The descending aorta was carefully perfused directly in its thoracic segment. The vessel was cannulated and physiological saline was injected into the arterial system.

For the study of the arterial system, the descending aorta of six birds were injected with India ink, and for the venous system, another six birds were injected with India ink through the caudal vena cava.

Tissue blocks of the phallus were prepared for light microscopy, selected areas were removed, trimmed into small blocks and immersion-fixed in $10 \%$ buffered formalin for a minimum period of 48 hours. The tissue blocks were conventionally processed for light microscopy, and histological sections were stained with haematoxylin and eosin $(\mathrm{H} \& \mathrm{E})$. The distribution of the arteries and veins was studied and described using an Olympus $\mathrm{BH}-2$ microscope and appropriate information was digitally recorded with a Nikon Coolpix 4500 digital camera. The diameter of the vessels 
was determined with the aid of a calibrated stage micrometer (Graticules Pyser-SGI Ltd., UK).

For transmission electron microscopy (TEM) the vascular system of the reproductive tract was flushed free of blood, in six birds, as described above. Small blocks of tissue were removed from the phallus and the sponge-like tissue (consisting of numerous lymph spaces traversed by connective tissue septa or struts), immersed in $2.5 \%$ glutaraldehyde in $0.1 \mathrm{M}$ cacodylate buffer ( $\mathrm{pH}$ 7.4). The specimens were stored at $4{ }^{\circ} \mathrm{C}$ prior to processing for TEM. In the lab the tissue blocks were conventionally processed for TEM.

\section{RESULTS}

\section{General morphological features}

The phallus, was attached to the ventral wall of the cloaca, it comprised of a base and a conical shaft. In general, the phallus comprises two fibroelastic bodies, one phallic sulcus, one elastic vascular body and one pair of muscles.

The phallus was lined externally by stratified squamous epithelium, and its root was surrounded ventro-laterally by a sponge-like tissue (Figs. 1, 2). The walls of the phallic sulcus were composed of erectile tissue, they were lined externally by stratified squamous epithelium (Fig. 3). The phallic sulcus was dorsally situated, it originated close to the papilla of deferent duct and extended until the tip of the organ.

\section{Observations of blood vessels distribution}

The naming of blood vessels (blood capillaries, arterioles, arteries, venules and collecting veins) was done according to the dimensional criteria supplied by Rhodini [16]. The root of the phallus was surrounded ventro-laterally by a sponge-like structure consisting of numerous large lymph spaces traversed by connective tissue septa or struts. The connective tissue struts were richly supplied with capillaries, relatively large arterial vessels (Fig. 1) as well as abundant venules and large collecting veins (Fig. 2).

The connective tissue septa were randomly arranged and varied in size and shape, but all were characterised by an extensive network of blood vessels as indicated above. The variably sized intervening lymphatic spaces communicated with each other forming an extensive lymphatic reservoir.

The walls of the phallic sulcus were composed of closely packed, regularly spaced mucosal folds covered by a strati- fied squamous epithelium. The surface epithelium was supported by a relatively wide layer of connective tissue characterised by an extensive network of small lymph spaces. This well-developed layer of erectile tissue displayed a zone of smaller blood vessels, mainly capillaries, lying beneath the epithelium, whereas larger, randomly distributed arterioles and a few sparsely distributed venules were scattered throughout (Fig. 3). When compared to the spongy tissue at the root of the phallus, the erectile tissue lining the phallic sulcus presented relatively few capillaries and venules. Numerous lymphocytes were also a feature of this tissue. The lymph spaces communicated with larger spaces situated in a deeper zone of connective tissue. This zone resembled some of the features of the spongy tissue described above, although the septa were more substantial, forming a greater proportion of the tissue. Numerous capillaries, arterioles and some larger arterial vessels were prominent within the septa. Prominent accumulations of colloid-like substance were obvious in the larger lymph spaces indicating the presence of lymph (Fig. 3).

The elastic vascular body located in the ventral part of the body of the phallus, exhibited a few arterial vessels (arterioles and capillaries) that were sparsely distributed in the elastic connective tissue. The elastic vascular body was continuous with the fibrous body which was composed of collagen fibres and poorly vascularised. However, it did demonstrate an erectile potential by virtue of a low incidence of lymphatic channels. The fibrous body did not exhibit blood vessels and contained collagen fibres, a few lymphatic channels (some of which contained lymph) and scant erectile tissue.

\section{Ultrastructural features}

The capillaries located in the connective tissue struts forming the spongy tissue at the base of the phallus (Figs. 4 B, C) displayed both wide and attenuated endothelial profiles. The most obvious morphological feature was the presence of numerous wide fenestrations beneath which the basal lamina remained continuous (Fig. 4 C). A diaphragm sealing the fenestrations could not be convincingly demonstrated in the material studied. Numerous cytoplasmic processes extended into the lumen from the surface of the endothelium. In certain areas the cytoplasmic processes appeared to enclose and sequester luminal contents. During this process the cytoplasmic extensions trapped material from the lumen and interiorized it into the endothelium, 


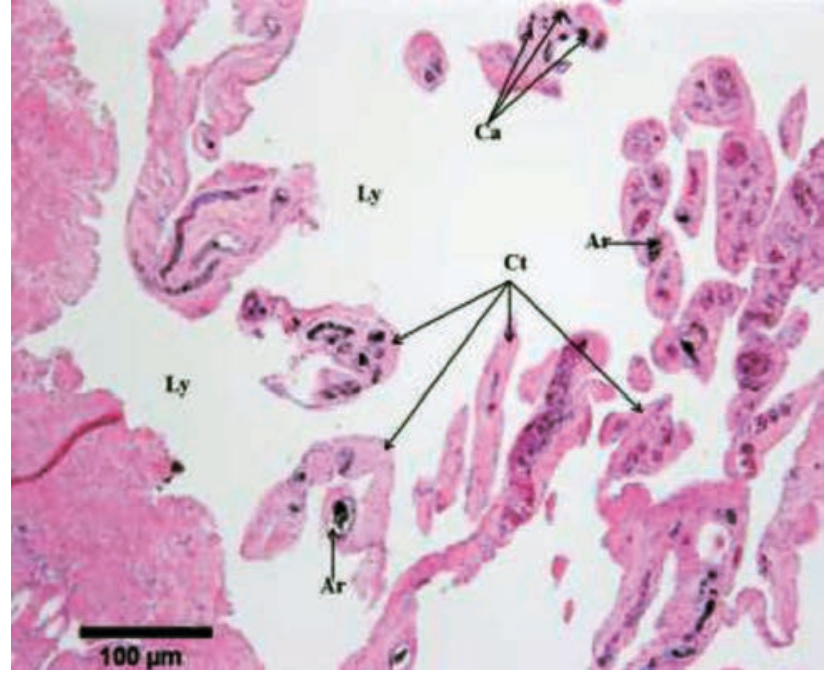

Fig. 1. Light micrograph of the spongy tissue at the root of the phallus. Note the variably sized connective tissue struts $(\mathrm{Ct})$ with blood capillaries (Ca) and arterioles (Ar) filled with India ink. There are empty lymph spaces (Ly) between the struts

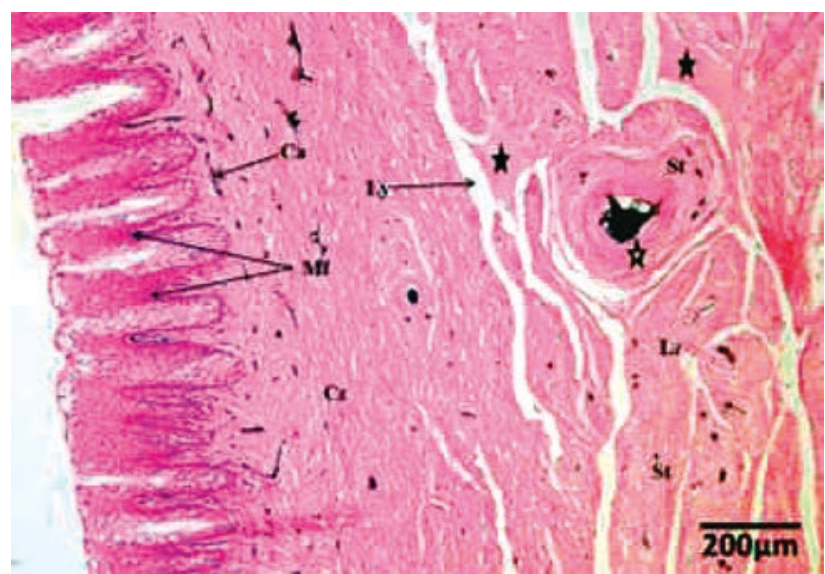

Fig. 3. Light micrograph of the phallic sulcus. There are regular mucosal folds (Mf) lining the surface, the more compact zone $(\mathrm{Cz})$ with scattered capillaries $(\mathrm{Ca})$ and the looser zone $(\mathrm{Lz})$ with connective tissue struts (St) containing larger vessels (white star) as well as capillaries. Colloid-like substance (black stars) lies in the lymph spaces (Ly) between the struts. India ink injection.pinocytotic vesicles (stars), lumen $(\mathrm{Lu})$, basal lamina (Bl)

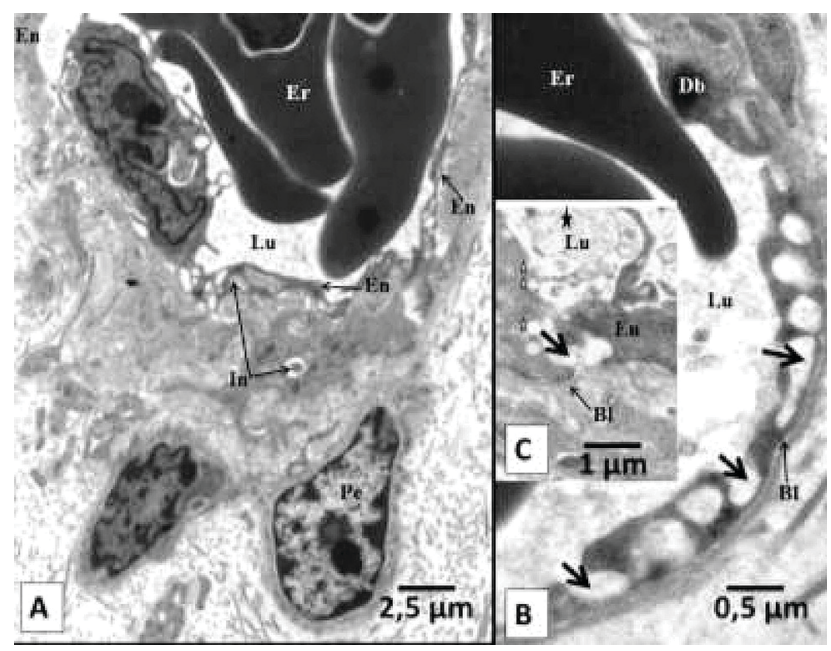

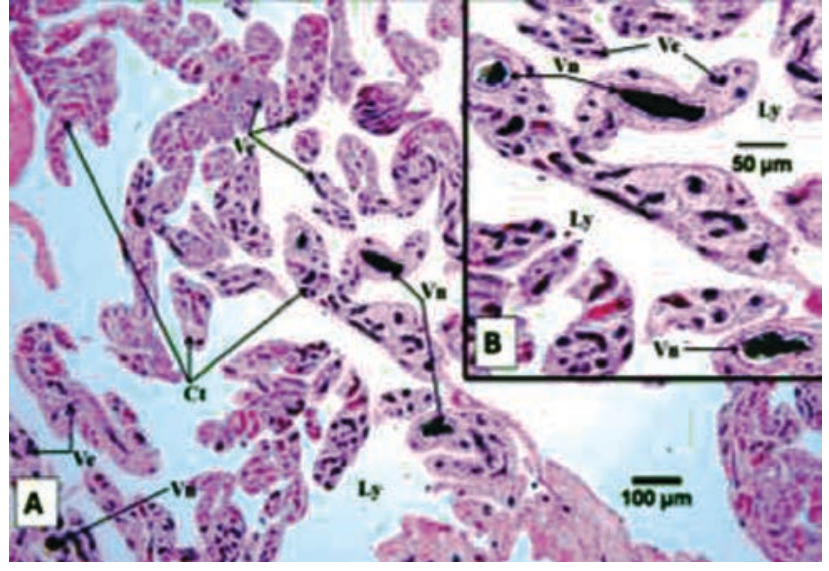

Fig. 2. A) Region similar to that shown in Fig. 5. but with India ink injected into the venous system. There are connective tissue struts $(\mathrm{Ct})$ with abundant venules ( $\mathrm{Ve}$ ) and collecting veins ( $\mathrm{Vn}$ ) and the large intervening lymph spaces (Ly). B) Magnification of part of Fig. A) showing tissue struts with venules (Ve) and collecting veins (Vn). There are empty lymph spaces between the struts (Ly)

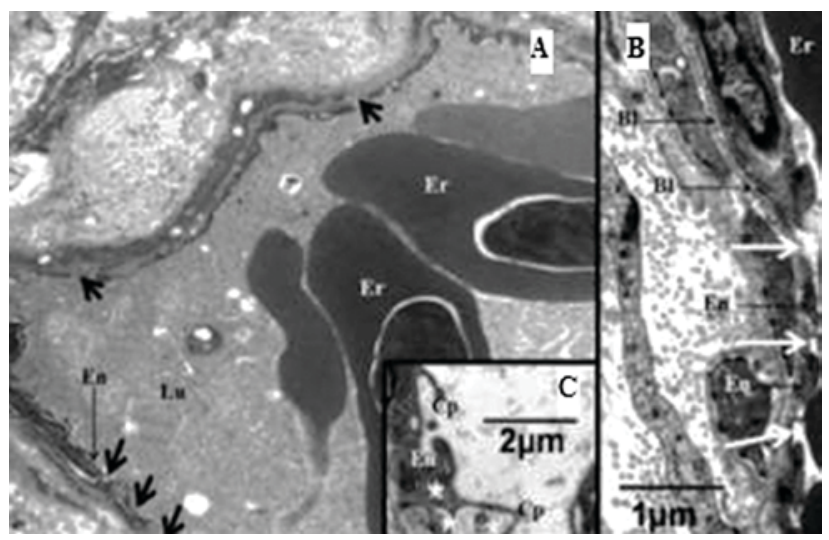

Fig.4. A) Venule in the spongy tissue at the root of the phallus displaying wide fenestrations (thick arrows) in the endothelium (En). Erythrocytes (Er) are packed in the vessel lumen (Lu). B) Longitudinal section of a capillary in the spongy tissue. The endothelium (En) is discontinued by wide fenestrations (white arrows) although the basal lamina (Bl) is continuous. C) A blood capillary displaying cytoplasmic processes $(\mathrm{Cp})$ extending from the vessel wall (En) and which appear to be sequestrating luminal contents (stars)

Fig. 5. A) Electron micrograph of a blood capillary in the erectile tissue of the phallic sulcus demonstrating complex interdigitations of the endothelium (En). Internalised material (In), lumen (Lu), erythrocytes (Er), presumptive pericyte $(\mathrm{Pe})$. B) Higher magnification of part of the capillary wall in Fig. $2 \mathrm{~A}$. There is apparent internalisation of luminal contents resulting in narrow strips of attenuated endothelium (short thick arrows) resting on a continuous basal lamina (Bl). Dense body (Db), lumen (Lu), erythrocytes (Er). Inset C) Higher magnification of the capillary endothelium (En) showing cytoplasmic processes in the act of internalising luminal material (black star). An endothelial fenestration (short thick arrow) is apparent as well as formation of pinocytotic vesicles (stars), lumen ( $\mathrm{Lu})$, basal lamina (Bl) 
resulting in the formation of pale vesicle-like structures. The endothelium was extremely irregular in profile, and in some instances was composed of more than one layer of overlapping squamous cells joined by apical adhering junctions. In addition to the vesicles, the endothelium displayed mitochondria, lysosomes, profiles of RER and free ribosomes. Arterioles were structurally similar in appearance to the capillaries but also revealed dense bodies. Internalisation of luminal material was also observed.

Venules were generally lined by an attenuated endothelium which was broken in places by wide fenestrations (Fig. 4 A). A continuous basal lamina was again obvious. The endothelial cells displayed blunt luminal projections and regions of cellular overlap were marked by sparse adhering junctions. In addition to the standard cytoplasmic organelles, the cells also displayed occasional dense bodies as well as pinocytotic vesicles.

The vessels present in the erectile tissue lining the phallic sulcus exhibited features similar to those seen in the spongy tissue in respect of their basic structure and organelle content. However, the endothelial cells were markedly more intricate and irregular in design, displaying numerous interdigitating cytoplasmic processes, particularly in regions of overlapping cells. The capillary endothelium displayed numerous fenestrations as well as regions where apparent sequestration of luminal contents resulted in exceptionally attenuated stretches of endothelial cytoplasm. In both instances a well-developed, continuous basal lamina was apparent (Fig. 5 A, B, C).

\section{DISCUSSION}

A brief overview based on the available literature and on the histological observations from the present study is presented to provide proper perspective on the distribution of blood vessels in the phallus. This study provides the first information on the distribution and structure of the microvasculature of the ostrich phallus. The specialized morphology (gaps and fenestrations, irregular endothelium, internalization of luminal material) of vessels in the spongy and erectile tissue of the phallus are previously unreported features which appear to be consistent with the erection mechanism reported in the ostrich.

The spongy tissue that lies ventro-lateral to the root of the phallus in the ostrich is comparable to a concentration of tissue with similar structural features that has been referred to as the paracloacal vascular body in various avian species. This spongy tissue in the ostrich has been termed the "great erectile cushion" [8], "lymphobulbus phallus" [15] and "lymphobulbus phalli" in Nomina Anatomica Avium [9].

The paracloacal vascular body is considered to be an accessory organ of the phallus. It develops at puberty and transmits lymph from arterial vessels into the phallus, during tumescence [6]. Earlier authors suspected the presence of the paracloacal vascular body in the ostrich [8], but they failed to provide adequate evidence of its existence. This report therefore is the first to localize, describe the gross and histological features of this organ, as well as describe its microvasculature.

In the rooster, the paracloacal vascular body is a small, red, oval structure located in the wall of the urodeum, close to the receptacle of the deferent duct and is covered by a connective tissue capsule [12]. The sponge-like tissue was supplied by branches of the pudendal artery and drained by branches of the pudendal vein $[3,4]$.

When viewed by light microscopy, the paraclocal vascular body reveals a capsule, trabeculae, capillary cords and lymphatic spaces. The lymphatic spaces are divided into peripheral lymphatic spaces, under the capsule, and internal lymphatic spaces in the parenchyma [19]. The lymphatic channels or spaces are present close to groups of blood capillaries [7]. The trabeculae contain one or two arterioles and venules. These features were verified in the present study of the spongy tissue of the ostrich phallus, except that the connective tissue element was not obviously divided into capillary cords and trabeculae. In the ostrich, larger blood vessels were found together with capillaries. Scanning electron microscopy (SEM) of corrosion casts of the paracloacal vascular body confirmed the existence of anastomosing vascular cords, between which were lymphatic spaces [19].

The basic structure of the paracloacal body, namely, lymph-filled spaces separated by vascular-rich fibrous cords as described in the fowl $[7,19]$ has also been confirmed in the turkey [10] and in the guinea fowl [17]. Although Sugimura et al. [19] and Sasaki et al. [17] observed only a few fenestrations in the blood capillary endothelium of the fowl and guinea fowl respectively, Gunawardana and Scott [7] described numerous fenestrations without closing membranes in the rooster. A similar situation was observed in the ostrich therefore supporting a previously held view [11] that fluid leaves the 
capillaries by diffusion rather than by active transport. It should be conceded, however, that the large numbers of pinocytotic vesicles noted in the ostrich blood vessels certainly suggests that active transport of fluid may play a supplementary role.

The specific morphological features of the spongy tissue described in this study, as well as the particular distribution of abundant, variably-sized capillaries and venules with fenestrations, would suggest that this structure participates in the mechanism of erection of the phallus in the ostrich, as has been demonstrated in other avian species $[6,7,12$, $13,17,20]$. That the spongy tissue at the root of the ostrich phallus supplies the fluids for erection is based on the presence within the spongy tissue (lymphobulbus phalli) of lymphatic vessels (lymph channels), as well as connective tissue strands with arterial vessels (capillaries and arterioles) and venous vessels (venules and collecting veins) that are richly supplied with fenestrations.

This study therefore confirms the existence of a paravascular cloacal body in the ostrich, and also that the mechanism of erection of the phallus in the ostrich is lymphatic as previously reported by Rautenfeld [15]. The use of the term "lymphobulbus phalli" in respect of the ostrich phallus [9] is thus supported.

The erectile tissue in the sulcus of the phallus exhibits the same morphological features as that of the lymphobulbus phalli, although in the latter, this tissue is more abundant, more complex, and is the main source of lymph for phallic erection in the ostrich. Additionally, there was no indication that the erectile tissue in the sulcus phalli communicates with the elastic vascular body that lies ventrocaudal to the shaft of the phallus. In the present study, the elastic vascular body did not reveal much erectile tissue, in contrast to earlier observations that the elastic vascular body contains an inner core of erectile tissue [8].

\section{CONCLUSIONS}

In conclusion, this study demonstrated that the lymphobulbus phalli and erectile tissue of the phallic sulcus, the presence of many fenestrations in the capillaries, arterioles and venules reflect the potential for rapid fluid exchange consistent with the process of erection and detumescence. Additionally, the relatively loose arrangement of overlapping endothelial cells connected by only a few adhering junctions in these vessels would also assist in the rapid drainage of lymph from the lymph spaces to the blood circulation during detumescence.

\section{REFERENCES}

1. Aire, T. A., 2007: Spermatogenesis and testicular cycles. In Jamieson, B. M. G. (Ed.): Reproductive Biology and Phylogeny of Birds. Science Publishers, Enfield, New Hampshire, 279-347.

2. Duerden, J. E., 1912: The anatomy and physiology of the ostrich. C - The internal organs. South African Agricultural Journal, April/May, 1-27.

3. Elias, M.Z. J., Aire, T.A., Soley, J.T., 2007: Macroscopic features of arterial supply to the reproductive system of the male ostrich (Struthio camelus). Anat. Histol. Embryol., 36, 255-262.

4. Elias, M.Z. J., Aire, T. A., Soley, J. T., 2008: Macroscopic features of the venous drainage of the reproductive system of the male ostrich (Struthio camelus). Onderst. J. Vet. Res., 75, 289-298.

5. Fowler, M.E., 1991: Comparative clinical anatomy of ratites. J. Zoo Wildlife Med., 22, 204-227.

6. Fujihara, N., Nishiyama, H., Nakashima, N., 1976: Studies on the accessory reproductive organs in the drake. 2. Macroscopic and microscopic observations on the cloaca of the drake with special reference to the ejaculatory groove. Poult. Sci., 55, 927-935.

7. Gunawardana, V.K., Scott, M. G., 1978: On the structure of the vascular body in the domestic fowl. Journal of Anatomy, 127, 447-457.

8. King, A.S., 1981: Form and Function in Birds. Academic Press, London, 459 pp.

9. King, A.S., 1993: Apparatus urogenitalis (Systema urogenitale). In Baumel, J. J. (Ed.): Handbook of Avian Anatomy. Nomina Anatomica Avium, Academic Press, Cambridge, 329-390.

10. Knight, C. E., 1970: The Anatomy of Structures Involved in the Erection-dilution Mechanism in the Male Domestic Fowl. Dissertation, Michigan State University, 129 pp.

11. Knight, C.E., 1984: Anatomy of the corpus vasculare paracloacale of the male turkey. Poult. Sci., 63, 1883-1891.

12. Kudo, N., Sugimura, M., Yamano, S., 1975: Anatomical studies of corpus paracloacalis vascularis in cocks. Jap. J. Vet. Res., $23,1-10$.

13. Lake, P.E., 1957: The male reproductive tract of the fowl. J. Anat., 91, 116-129.

14. Montgomerie, R., Briskie, J.V., 2007: Anatomy and evolution of copulatory structures. In Jamieson, B. M. G. (Ed.): 
Reproductive Biology and Phylogeny of Birds. Science Publishers, Enfield, New Hampshire, 115-178.

15. Rautenfeld, D.B., 1977: Mitteilungen zur künstlichen Besamung, Geschlecht Und Altersbestimmung beim Strauß (Struthio camelus australis, Gurney). Der Praktische Tierarzt, 5, 359-364.

16. Rhodini, J. A. G., 1974: Histology. A Textbook and Atlas. Oxford University Press, New York, 803 pp.

17. Sasaki, H., Nishida, T., Fujimura, H., Mochizuki, K., 1984: Vascular system of Paracloacal vascular body in the Guinea Fowl (Numida meleagris). Jap. J. Vet. Sci., 46, 425-435.
18. Soley, J. T., Elias, M.Z. J., Aire, T. A., 2007: Variations in gross pattern of venous drainage of the ostrich male reproductive tract: A comparison with the general avian model. In Proceedings of the 1st Conjoint International Conference on Fertility, Anatomy and Morphological Sciences. Lagos, Nigeria, 35-36.

19. Sugimura, M., Kudo, N., Yamano, S., 1975: Fine structures of corpus paracloacalis in cocks. Jap. J. Vet. Res., 23, 11-16.

20. Yamano, S., Sugimura, M., Kudo, N., 1977: The lymphatic system of the corpus paracloacalis vascularis and the second fold in the male domestic fowl. Jap. J. Vet. Res., 25, 93-98.

Received May 18, 2016 